\title{
Editorial: Ion Channels: Therapeutic Targets for Neurological Disease
}

\begin{abstract}
Panpan $\mathrm{Hou}^{1,2 *}$, Xiaona $\mathrm{Du}^{3,4,5 *}$ and Hailong $\mathrm{An}^{6 *}$
${ }^{1}$ Dr. Neher's Biophysics Laboratory for Innovative Drug Discovery, Macau University of Science and Technology, Taipa, Macao SAR, China, ${ }^{2}$ State Key Laboratory of Quality Research in Chinese Medicine, Macau University of Science and Technology, Taipa, Macao SAR, China, ${ }^{3}$ Department of Pharmacology, Hebei Medical University, Shijiazhuang, China, ${ }^{4}$ The Key Laboratory of Neural and Vascular Biology, Ministry of Education, Hebei Medical University, Shijiazhuang, China, ${ }^{5}$ The Key Laboratory of New Drug Pharmacology and Toxicology, Hebei Medical University, Shijiazhuang, China, ${ }^{6}$ Key Laboratory of Molecular Biophysics of Hebei, Institute of Biophysics, School of Science, Hebei University of Technology, Tianjin, China
\end{abstract}

Keywords: ion channels, drug target, neurological disease, single nucleotide polymorphism (SNP), hearing loss, spinal cord injury (SCI), schizophrenia, epilepsy

\section{Editorial on the Research Topic}

\section{Ion Channels: Therapeutic Targets for Neurological Disease}

\section{OPEN ACCESS}

Edited and reviewed by: Jean-Marc Taymans, Institut National de la Santé et de la Recherche Médicale (INSERM), France

*Correspondence:

Panpan Hou

pphou@must.edu.mo Xiaona $\mathrm{Du}$

duxiaona@hebmu.edu.cn Hailong An

hailong_an@hebut.edu.cn

Specialty section:

This article was submitted to Molecular Signalling and Pathways, a section of the journal Frontiers in Molecular Neuroscience

Received: 18 October 2021

Accepted: 25 October 2021

Published: 16 November 2021

Citation: Hou P, Du X and An H (2021) Editorial: Ion Channels: Therapeutic Targets for

Neurological Disease.

Front. Mol. Neurosci. 14:797327.

doi: 10.3389/fnmol.2021.797327
Contributing $11.6 \%$ of global DALYs (disability-adjusted life-years) and $16.5 \%$ of deaths from all causes, neurological disorders remain the leading cause of DALYs and the second leading cause of deaths (following cardiovascular diseases) over the world (Feigin et al., 2019). Neurological disorders are public health challenges that cause not only a decline in the quality of life for patients, but also a substantial burden for every family (Feigin et al., 2020). The prevalence of most neurological disorders increases with age, and the lack of effective treatment options continues to increase the number of patients (Feigin et al., 2019). Therefore, developing new strategies to prevent and treat the major neurological disorders is of great importance to improve human health.

Neurons communicate via rapid electrical activities that allow the nervous system to coordinate sensation, behavior, and emotion. Ion channels in neurons are the main information carrier of these neuronal electrical activities. They form conduction pores to allow selected ions $\left(\mathrm{Na}^{+}, \mathrm{K}^{+}\right.$, $\mathrm{Ca}^{2+}, \mathrm{Cl}^{-}$, etc.) to pass through the cell membrane and generate electrical signals for establishing the resting membrane potential (Du et al., 2014), shaping each phase of action potentials, and controlling the $\mathrm{Ca}^{2+}$ signaling etc. (Hou et al., 2016; Chiamvimonvat et al., 2017; Shi et al., 2021). Dysfunction of ion channels by inherited mutations, pathological changes, or unwanted druginduced side effects can alter ion flux across the membrane and cause neurological disorders. On the other hand, tremendous pre-clinical and clinical studies suggest that regulating the function of key ion channels involved in diseases can effectively alleviate the symptoms, supporting that ion channels are promising targets for treating neurological disorders.

In this topic named "Ion Channels: Therapeutic Targets for Neurological Disease," we collect and summarize the most recent studies including four original articles and two reviews focusing on ion channel modulation mechanisms and ion channels as potential targets for treating hearing loss, pain, spinal cord injury, ischemia-reperfusion injury, and schizophrenia.

Single nucleotide polymorphisms (SNPs) refers to DNA sequence polymorphisms caused by mutation of a single nucleotide. It is the most common form of genetic variants in humans, accounting for more than $90 \%$ of all known polymorphisms (International HapMap et al., 2007). Studies indicate that SNPs are associated with the severity of 
illness, such as Alzheimer's disease (Burns et al., 2011). SNPs are also common in ion channels. Plante et al. examined the effects of several non-synonymous SNPs on the KCNMA1 gene encoding the large conductance potassium (BK) channel (Atkinson et al., 1991). It turned out these SNPs can induce gain-of-function or loss-of-function effects on BK channels. For example, the SNP R800W slows down the activation rate, right shifts the conductance-voltage relation, and increases the amplitude of action potential-evoked currents. These loss-of-function effects are also conserved in the epilepsy-associated mutation D434G channel background. Overall, Plante et al. set a clear example that SNPs can modulate the function of BK channels. More basic and translational studies (for example the drug sensitivity change of SNPs) can be done to further understand the phenotypic and functional consequences of SNPs in ion channels.

The low-voltage activated (LVA) T-type calcium channel Cav3.2 sets a low threshold for action potential firing in dorsal root ganglion (DRG) neurons and plays a key role in pain sensation (White et al., 1989; Cain and Snutch, 2010). Cav3.2 channel is regulated by different proteins (Zhang et al., 2013; Weiss and Zamponi, 2017), including the neuronal actin-binding protein Kelch-like 1 (KLHL1; Aromolaran et al., 2009, 2010). Using KLHL1 knockout (KO) or knock-down mice models, Martínez-Hernández et al. found that KLHL1 is important for maintaining the $\mathrm{Ca}_{V} 3.2$ channel expression in DRG neurons. The $\mathrm{Ca}_{V} 3.2$ protein level in KLHL1 $\mathrm{KO}$ mice is specifically decreased, and the T-type current is smaller than the wild type without significant change in the voltage-dependent activation. These results clearly demonstrate that by modulating the $\mathrm{Ca}_{\mathrm{v}} 3.2$ channel expression, KLHL1 can regulate the DRG excitability and pain sensitivity, providing a potential target to treat peripheral pain.

Unlike voltage-gated ion channels that sense the membrane voltage change via the voltage sensing domain (Wang et al., 2017; Hou et al., 2020), the transient receptor potential channels (TRP channels) are ligand-gated ion channels that are sensitive to physical (heat, $\mathrm{pH}$, osmolarity, etc.) and chemical (ligands, $\mathrm{Ca}^{2+}$, etc.) stimuli from the environment, and therefore are important sensors for perception (Clapham, 2003). TRPV channels (the vanilloid subtype) are widely expressed and critical to nociception (Julius, 2013). TRPV3 is highly expressed in the cochlea hair cells, colocalizing with TRPV4 (Ishibashi et al., 2008). Studies on TRPV3KO mice model by Wang et al. found that a significant portion (27.7\%) showed impaired hearing associated with loss of cochlear hear cells, but most TRPV3KO mice $(72.3 \%)$ had normal hearing. Interestingly, compensatory upregulation of TRPV4 was observed in the TRPV3KO mice with normal hearing, suggesting that both TRPV4 and TRPV3 channels are important for maintaining normal hearing. Overall, these results offer not only novel insight into the molecular basis of hearing but also potential target for treating hearing loss.

The $\mathrm{Ca}^{2+}$ ion is also a key player for spinal cord injury (SCI), a seriously debilitating event that can lead to paralysis and even death (Ditunno and Formal, 1994). After SCI, damaged neurons release high concentrations of the neurotransmitter glutamate (Park et al., 2004), resulting in excessive intracellular $\mathrm{Ca}^{2+}$ and increasing cell death. To facilitate functional recovery after SCI, the combination of ion channel inhibitors to block the three main $\mathrm{Ca}^{2+}$ channels [Lom: for voltage-gated $\mathrm{Ca}^{2+}$ channels (Sattler et al., 1996), oxATP: for $\mathrm{P}_{2} \mathrm{X}_{7}$ receptors (Hollmann et al., 1991), and YM872: for $\mathrm{Ca}^{2+}$ permeable AMPA receptors (Hollmann et al., 1991)] has been proven an effective treatment for neurotrauma (O’Hare Doig et al., 2016, 2017). O'Hare Doig et al. further investigated this combinational strategy on a clinically relevant model of SCI and observed significant positive changes in early functional recovery and pathophysiology. Future studies on the combination of these ion channel blockers in chronic models of SCI are required to evaluate its long-term effect of treatment. Overall, O'Hare Doig et al. showed that the combination of $\mathrm{Ca}^{2+}$ channel blockers can be a promising strategy for treating SCI.

Cerebral ischemia-reperfusion injury (CIRI) can cause severe damage to the brain. During cerebral ischemia-reperfusion, oxidative stress occurs and a large number of inflammatory cytokines are present in the ischemic focus (Lin et al., 2016). $\mathrm{Wu}$ et al. summarized current studies about the roles of oxidative stress and inflammation in CIRI, as well as important signaling pathways and therapeutic options for CIRI. Similar to the abovementioned combination strategy works better for SCI than using individual blockers (Savigni et al., 2013), single antioxidant therapy can only reduce cerebral ischemic injury to a certain extent, and so far there is no simple strategy to control the neuroinflammation due to the complex mechanisms of inflammation in CIRI. To this point, Wu et al. proposed that future studies can focus on the development of a free radical scavenger with multiple mechanisms of action and the combination of free radical scavengers with antiinflammatory drugs.

Schizophrenia is a serious mental illness that affects $\sim 1 \%$ of the world population and places a major socio-economic burden (Blot et al., 2013). The World Health Organization (WHO) estimated that, in Western countries, direct annual costs of schizophrenia range from 1.6 to $2.6 \%$ of total health care expenditures. In the US alone, the annual economic burden of schizophrenia is more than US\$60 billion (Chong et al., 2016). The N-methyl-D-aspartate receptor (NMDAR) is important for the development of the nervous system and the formation of neuronal circuits (Moghaddam et al., 1997; Fellin et al., 2009), and accumulating evidence in human and animal studies support that NMDAR hypofunction is a convergence point of various symptoms of schizophrenia. Lee and Zhou summarized various animal models of NMDAR hypofunction generated by both pharmacological and genetic approaches, and how they relate to the pathophysiology of schizophrenia. Lee and Zhou also discussed limitations of these animal models and their potential utility for therapeutic applications. With the recent breakthrough on structural and pharmacological studies of NMDAR (Zhang et al., 2021), these animal models will provide useful platforms to identify novel therapeutics for schizophrenia.

Taken together, neurological disorder is one of the leading cause of deaths all over the world. This research topic highlights exciting new advances in the modulation mechanism of key 
ion channels in the nervous system, and the critical roles of ion channels in maintaining the physiological function and alleviating the symptoms of diseases. Further basic and clinical studies on ion channels will help develop new therapeutics for treating neurological disorders and improving human health.

\section{AUTHOR CONTRIBUTIONS}

All authors contributed equally to the writing and editing of the manuscript.

\section{REFERENCES}

Aromolaran, K. A., Benzow, K. A., Cribbs, L. L., Koob, M. D., and PiedrasRenteria, E. S. (2009). Kelch-like 1 protein upregulates T-type currents by an actin-F dependent increase in alpha $(1 \mathrm{H})$ channels via the recycling endosome. Channels 3, 402-412. doi: 10.4161/chan.3.6.9858

Aromolaran, K. A., Benzow, K. A., Cribbs, L. L., Koob, M. D., and Piedras-Renteria, E. S. (2010). T-type current modulation by the actin-binding protein Kelch-like 1. Am. J. Physiol. Cell Physiol. 298, C1353-1362. doi: 10.1152/ajpcell.00235.2009

Atkinson, N. S., Robertson, G. A., and Ganetzky, B. (1991). A component of calcium-activated potassium channels encoded by the Drosophila slo locus. Science 253, 551-555. doi: 10.1126/science. 1857984

Blot, K., Bai, J., and Otani, S. (2013). The effect of non-competitive NMDA receptor antagonist MK-801 on neuronal activity in rodent prefrontal cortex: an animal model for cognitive symptoms of schizophrenia. J. Physiol. 107, 448-451. doi: 10.1016/j.jphysparis.2013.04.003

Burns, L. C., Minster, R. L., Demirci, F. Y., Barmada, M. M., Ganguli, M., Lopez, O. L., et al. (2011). Replication study of genome-wide associated SNPs with late-onset Alzheimer's disease. Am. J. Med. Genet. B Neuropsychiatr. Genet. Off. Publ. Int. Soc. Psychiatr. Genet. 156B, 507-512. doi: 10.1002/ajmg.b.31194

Cain, S. M., and Snutch, T. P. (2010). Contributions of T-type calcium channel isoforms to neuronal firing. Channels 4, 475-482. doi: 10.4161/chan.4.6.14106

Chiamvimonvat, N., Chen-Izu, Y., Clancy, C. E., Deschenes, I., Dobrev, D., Heijman, J., et al. (2017). Potassium currents in the heart: functional roles in repolarization, arrhythmia and therapeutics. J. Physiol. 595, 2229-2252. doi: 10.1113/JP272883

Chong, H. Y., Teoh, S. L., Wu, D. B., Kotirum, S., Chiou, C. F., and Chaiyakunapruk, N. (2016). Global economic burden of schizophrenia: a systematic review. Neuropsychiatr. Dis. Treat. 12, 357-373. doi: 10.2147/NDT.S96649

Clapham, D. E. (2003). TRP channels as cellular sensors. Nature 426, 517-524. doi: $10.1038 /$ nature 02196

Ditunno, J. F. Jr., and Formal, C. S. (1994). Chronic spinal cord injury. N. Engl. J. Med. 330, 550-556. doi: 10.1056/NEJM199402243300808

Du, X., Hao, H., Gigout, S., Huang, D., Yang, Y., Li, L., et al. (2014). Control of somatic membrane potential in nociceptive neurons and its implications for peripheral nociceptive transmission. Pain 155, 2306-2322. doi: 10.1016/j.pain.2014.08.025

Feigin, V. L., Abajobir, A. A., Abate, K. H., Abd-Allah, F., Abdulle, A. M., Abera, S. F., et al. (2019). Global, regional, and national burden of neurological disorders, 1990-2016: a systematic analysis for the Global Burden of Disease Study 2016. Lancet Neurol. 18, 459-480. doi: 10.1016/S1474-4422(18)30499-X

Feigin, V. L., Vos, T., Nichols, E., Owolabi, M. O., Carroll, W. M., Dichgans, M., et al. (2020). The global burden of neurological disorders: translating evidence into policy. Lancet Neurol. 19, 255-265. doi: 10.1016/S1474-4422(19) 30411-9

Fellin, T., Halassa, M. M., Terunuma, M., Succol, F., Takano, H., Frank, M., et al. (2009). Endogenous nonneuronal modulators of synaptic transmission control cortical slow oscillations in vivo. Proc. Natl. Acad. Sci. U. S. A. 106, 15037-15042. doi: 10.1073/pnas.0906419106

Hollmann, M., Hartley, M., and Heinemann, S. (1991). $\mathrm{Ca}^{2+}$ permeability of KAAMPA-gated glutamate receptor channels depends on subunit composition. Science 252, 851-853. doi: 10.1126/science.1709304

\section{FUNDING}

This work was supported by the National Natural Science Foundation of China (Nos. 32171221 to $\mathrm{PH}, 81870872$ to $\mathrm{XD}$, and 81830061 to $\mathrm{HA})$, the Natural Science Foundation of Tianjin of China (No. 19JCYBJC28300 to HA), and the Natural Science Foundation of Hebei Province of China (No. H2020202005 to HA). This work was also funded by Dr. Neher's Biophysics Laboratory for Innovative Drug Discovery (Grant no. 001/2020/ALC) as supported by the Macau Science and Technology Development Fund.

Hou, P., Kang, P. W., Kongmeneck, A. D., Yang, N. D., Liu, Y., Shi, J., et al. (2020) Two-stage electro-mechanical coupling of a KV channel in voltage-dependent activation. Nat. Commun. 11:676. doi: 10.1038/s41467-020-14406-w

Hou, P., Xiao, F., Liu, H., Yuchi, M., Zhang, G., Wu, Y., et al. (2016). Extrapolating microdomain $\mathrm{Ca}\left({ }^{2+}\right)$ dynamics using $\mathrm{BK}$ channels as a $\mathrm{Ca}\left({ }^{2+}\right)$ sensor. Sci. Rep. 6:17343. doi: $10.1038 /$ srep 17343

International HapMap, C., Frazer, K. A., Ballinger, D. G., Cox, D. R., Hinds, D. A., Stuve, L. L., et al. (2007). A second generation human haplotype map of over 3.1 million SNPs. Nature 449, 851-861. doi: 10.1038/nature06258

Ishibashi, T., Takumida, M., Akagi, N., Hirakawa, K., and Anniko, M. (2008). Expression of transient receptor potential vanilloid (TRPV) 1, 2, 3, and 4 in mouse inner ear. Acta Oto-laryngol. 128, 1286-1293. doi: $10.1080 / 00016480801938958$

Julius, D. (2013). TRP channels and pain. Ann. Rev. Cell Dev. Biol. 29, 355-384. doi: 10.1146/annurev-cellbio-101011-155833

Lin, L., Wang, X., and Yu, Z. (2016). Ischemia-reperfusion injury in the brain: mechanisms and potential therapeutic strategies. Biochem. Pharmacol. 5:213. doi: 10.4172/2167-0501.1000213

Moghaddam, B., Adams, B., Verma, A., and Daly, D. (1997). Activation of glutamatergic neurotransmission by ketamine: a novel step in the pathway from NMDA receptor blockade to dopaminergic and cognitive disruptions associated with the prefrontal cortex. J. Neurosci. Off. J. Soc. Neurosci. 17, 2921-2927. doi: 10.1523/JNEUROSCI.17-08-02921.1997

O’Hare Doig, R. L., Bartlett, C. A., Smith, N. M., Hodgetts, S. I., Dunlop, S. A., Hool, L., et al. (2016). Specific combinations of ion channel inhibitors reduce excessive $\mathrm{Ca}(2+)$ influx as a consequence of oxidative stress and increase neuronal and glial cell viability in vitro. Neuroscience 339, 450-462. doi: 10.1016/j.neuroscience.2016.10.005

O’Hare Doig, R. L., Chiha, W., Giacci, M. K., Yates, N. J., Bartlett, C. A., Smith, N. M., et al. (2017). Specific ion channels contribute to key elements of pathology during secondary degeneration following neurotrauma. BMC Neurosci. 18:62. doi: 10.1186/s12868-017-0380-1

Park, E., Velumian, A. A., and Fehlings, M. G. (2004). The role of excitotoxicity in secondary mechanisms of spinal cord injury: a review with an emphasis on the implications for white matter degeneration. J. Neurotr. 21, 754-774. doi: 10.1089/0897715041269641

Sattler, R., Tymianski, M., Feyaz, I., Hafner, M., and Tator, C. H. (1996). Voltagesensitive calcium channels mediate calcium entry into cultured mammalian sympathetic neurons following neurite transection. Brain Res. 719, 239-246. doi: 10.1016/0006-8993(96)00125-4

Savigni, D. L., O’Hare Doig, R. L., Szymanski, C. R., Bartlett, C. A., Lozic, I., Smith, N. M., et al. (2013). Three Ca2+ channel inhibitors in combination limit chronic secondary degeneration following neurotrauma. Neuropharmacology 75, 380-390. doi: 10.1016/j.neuropharm.2013.07.034

Shi, S., Pang, C., Ren, S., Sun, F., Ma, B., Guo, S., et al. (2021). Molecular dynamics simulation of TMEM16A channel: linking structure with gating. Biochim. Biophys. Acta Biomembr. 1864:183777. doi: 10.1016/j.bbamem.2021.183777

Wang, X., Chen, Y., Zhang, Y., Guo, S., Mo, L., An, H., et al. (2017). Eag1 voltage-dependent potassium channels: structure, electrophysiological characteristics, and function in cancer. J. Membr. Biol. 250, 123-132. doi: 10.1007/s00232-016-9944-8

Weiss, N., and Zamponi, G. W. (2017). Trafficking of neuronal calcium channels. Neuronal. Signal. 1:NS20160003. doi: 10.1042/NS20160003 
White, G., Lovinger, D. M., and Weight, F. F. (1989). Transient low-threshold $\mathrm{Ca}^{2+}$ current triggers burst firing through an afterdepolarizing potential in an adult mammalian neuron. Proc. Natl. Acad. Sci. U. S. A. 86, 6802-6806. doi: $10.1073 /$ pnas.86.17.6802

Zhang, Y., Jiang, X., Snutch, T. P., and Tao, J. (2013). Modulation of low-voltageactivated T-type $\mathrm{Ca}^{2+}$ ) channels. Biochim. Biophys. Acta 1828, 1550-1559. doi: 10.1016/j.bbamem.2012.08.032

Zhang, Y., Ye, F., Zhang, T., Lv, S., Zhou, L., Du, D., et al. (2021). Structural basis of ketamine action on human NMDA receptors. Nature 596, 301-305. doi: $10.1038 / s 41586-021-03769-9$

Conflict of Interest: The authors declare that the research was conducted in the absence of any commercial or financial relationships that could be construed as a potential conflict of interest.
Publisher's Note: All claims expressed in this article are solely those of the authors and do not necessarily represent those of their affiliated organizations, or those of the publisher, the editors and the reviewers. Any product that may be evaluated in this article, or claim that may be made by its manufacturer, is not guaranteed or endorsed by the publisher.

Copyright $\odot 2021 \mathrm{Hou}, \mathrm{Du}$ and An. This is an open-access article distributed under the terms of the Creative Commons Attribution License (CC BY). The use, distribution or reproduction in other forums is permitted, provided the original author(s) and the copyright owner(s) are credited and that the original publication in this journal is cited, in accordance with accepted academic practice. No use, distribution or reproduction is permitted which does not comply with these terms. 